\title{
Graph weights arising from Mayer and Ree-Hoover theories of virial expansions
}

\author{
Amel Kaouche and Pierre Leroux \\ LaCIM, Département de Mathématiques, Université du Québec à Montréal
}

\begin{abstract}
We study graph weights (i.e., graph invariants) which arise naturally in Mayer's theory and Ree-Hoover's theory of virial expansions in the context of a non-ideal gas. We give special attention to the Second Mayer weight $w_{M}(c)$ and the Ree-Hoover weight $w_{R H}(c)$ of a 2-connected graph $c$ which arise from the hard-core continuum gas in one dimension. These weights are computed using signed volumes of convex polytopes naturally associated with the graph $c$. Among our results are the values of Mayer's weight and Ree-Hoover's weight for all 2-connected graphs $b$ of size at most 8 , and explicit formulas for certain infinite families.

Résumé. Nous étudions les poids de graphes (c'est-à-dire, les invariants de graphes) qui apparaissent naturellement dans la théorie de Mayer et la théorie de Ree-Hoover pour le développement du viriel dans le contexte d'un gaz imparfait. Nous donnons une attention particulière au deuxième poids $w_{M}(c)$ de Mayer et au poids $w_{R H}(c)$ de ReeHoover d'un graphe 2-connexe $c$ dans le cas d'un gaz à noyaux durs et à positions continues en une dimension. Ces poids sont calculés à partir de volumes signés de polytopes covexes associés naturellement au graphe $c$. Parmi nos résultats sont les valeurs du poids de Mayer et du poids de Ree-Hoover pour tous les graphes 2-connexes $b$ de taille au plus 8 , et des formules explicites pour certaines familles infinies.
\end{abstract}

Keywords: graph invariants, non-ideal gas, hard sphere gas, Mayer weights, virial expansion.

\section{Introduction}

Graph weights can be defined as functions on (simple, finite) graphs taking scalar or polynomial values and which are invariant under isomorphism, i.e., under vertex relabelling. Since most graphical concepts share this invariance property, examples of graph weights abound. For instance, the graph complexity $\gamma(g)$ of a graph $g$, which is defined as the number of maximal spanning forests of $g$, is an example of a graph weight. In the context of a non-ideal gas in a vessel $V \subseteq \mathbb{R}^{d}$, the Second Mayer weight $w_{M}(c)$ of a connected graph $c$, over the set $[n]=\{1,2, \ldots, n\}$ of vertices, is defined by

$$
w_{M}(c)=\int_{\left(\mathbb{R}^{d}\right)^{n-1}} \prod_{\{i, j\} \in c} f\left(\left\|\overrightarrow{x_{i}}-\overrightarrow{x_{j}}\right\|\right) d \overrightarrow{x_{1}} \cdots d \overrightarrow{x_{n-1}}, \quad \overrightarrow{x_{n}}=0
$$

where $\overrightarrow{x_{1}}, \ldots, \overrightarrow{x_{n}}$ are variables in $\mathbb{R}^{d}$ representing the positions of $n$ particles in $V(V \rightarrow \infty),\left\|\overrightarrow{x_{i}}-\overrightarrow{x_{j}}\right\|$ means the euclidean distance in $\mathbb{R}^{d}$ between $\overrightarrow{x_{i}}$ and $\overrightarrow{x_{j}}$, the value $\overrightarrow{x_{n}}=0$ being arbitrarily fixed, and where $f=f(r)$ is a real-valued function associated with the pair-wise interaction potential of the particles. See 
[11, 4]. For example, in the case of a Gaussian interaction, where $f(r)=-\exp \left(-\alpha r^{2}\right), \alpha>0$, we have, for a connected graph $c$ with $n$ vertices and $e(c)$ edges, $w(c)=(-1)^{e(c)}\left(\frac{\pi}{\alpha}\right)^{\frac{d(n-1)}{2}} \gamma(c)^{-\frac{d}{2}}$, where $\gamma(c)$ is the graph complexity, which, in the case of a connected graph, is the number of spanning trees. The total sum of weights of connected graphs over $[n]$ is denoted by

$$
|\mathcal{C}[n]|_{w_{M}}=\sum_{c \in \mathcal{C}[n]} w_{M}(c) .
$$

The interest of this sum in statistical mechanics comes from the fact that the pressure $P$ of the system is given by it's exponential generating function as follows:

$$
\frac{P}{k T}=\mathcal{C}_{w_{M}}(z)=\sum_{n \geq 1}|\mathcal{C}[n]|_{w_{M}} \frac{z^{n}}{n !}
$$

where $k$ is a constant, $T$ is the temperature and $z$ is a variable called the fugacity or the activity of the system. It is known that the weight $w_{M}$ is multiplicative over 2-connected components so that it is sufficient to compute the weights $w_{M}(b)$ for 2-connected graphs $b \in \mathcal{B}[n]$ ( $\mathcal{B}$ for blocks). Moreover, these occur in the so-called virial expansion

$$
\frac{P}{k T}=\rho+\beta_{2} \rho^{2}+\beta_{3} \rho^{3}+\cdots,
$$

where $\rho$ is the density. Indeed, it can be shown that

$$
\beta_{n}=\frac{1-n}{n !}|\mathcal{B}[n]|_{w_{M}} .
$$

In order to compute this expansion numerically, Ree and Hoover [7] introduced a modified weight denoted by $w_{R H}(b)$, for 2-connected graphs $b$, which greatly simplifies the computations. It is defined by

$$
w_{R H}(b)=\int_{\left(\mathbb{R}^{d}\right)^{n-1}} \prod_{\{i, j\} \in b} f\left(\left\|\overrightarrow{x_{i}}-\overrightarrow{x_{j}}\right\|\right) \prod_{\{i, j\} \in \bar{b}} \vec{f}\left(\left\|\overrightarrow{x_{i}}-\overrightarrow{x_{j}}\right\|\right) d \overrightarrow{x_{1}} \cdots d \overrightarrow{x_{n-1}}, \quad \overrightarrow{x_{n}}=0,
$$

where $\bar{f}(r)=1+f(r)$ and $\bar{b}=K_{n} \backslash b$ is the complementary graph of $b$. Using this new weight, Ree and Hoover [7, 8, 9] and later Clisby and McCoy [1, 2] have computed the virial coefficients $\beta_{n}$, for $n$ up to 10 , in dimension $d \leq 8$, in the case of the hard core continuum gas, that is when the interaction is given by

$$
f(r)=-\chi(r<1), \quad \bar{f}(r)=\chi(r \geq 1) .
$$

While physicists are interested in summing the weights of all connected or 2-connected graphs of a given order, the present paper focuses on individual graph contributions and their combinatorial significance. In particular, we study the graph weights $w_{M}(b)$ and $w_{R H}(b)$ in the context of the hard core continuum gas, defined by $(7)$, in dimension $d=1$. Among our results are the values $w_{M}(c)$ and $w_{R H}(c)$ for all 2-connected graphs $c$ of size at most 8 (see [3]). Moreover, each 2-connected graph $c$ of size $N_{c}$, for which $w_{R H}(c) \neq 0$, of the form $c=K_{N_{c}} \backslash \bar{c}$, determines an infinite family of graphs $g_{n}=K_{n} \backslash \bar{c}$, $n \geq N_{c}$. Here we give explicit formulas for $w_{R H}\left(K_{n} \backslash \bar{c}\right)$, for all these infinite families with $4 \leq N_{c} \leq 7$, and give $w_{M}\left(K_{n} \backslash \bar{c}\right)$ for some of them. 


\section{The hard-core continuum gas in one dimension}

Consider $n$ hard particles of diameter 1 on a line segment. The hard-core constraint translates into the interaction potential $\varphi$, with $\varphi(r)=\infty$, if $r<1$, and $\varphi(r)=0$, if $r \geq 1$, and the Mayer function $f_{i j}$ and the Ree-Hoover function $\bar{f}_{i j}$ are defined by

$$
f_{i j}=-\chi\left(\left|x_{i}-x_{j}\right|<1\right), \quad \bar{f}_{i j}=1+f_{i j}=\chi\left(\left|x_{i}-x_{j}\right| \geq 1\right) .
$$

From [4], we can write the Mayer weight function $w_{M}(c)$ of a connected graph $c$ as

$$
w_{M}(c)=(-1)^{e(c)} \int_{\mathbb{R}^{n-1}} \prod_{\{i, j\} \in c} \chi\left(\left|x_{i}-x_{j}\right|<1\right) d x_{1} \ldots d x_{n-1}, \quad x_{n}=0,
$$

and the Ree-Hoover's weight function $w_{R H}(c)$ of a 2-connected graph $c$ as

$$
w_{R H}(c)=(-1)^{e(c)} \int_{\mathbb{R}^{n-1}} \prod_{\{i, j\} \in c} \chi\left(\left|x_{i}-x_{j}\right|<1\right) \prod_{\{i, j\} \in \bar{c}} \chi\left(\left|x_{i}-x_{j}\right|>1\right) d x_{1} \ldots d x_{n-1}, \quad x_{n}=0
$$

where $e(c)$ is the number of edges of $c$ and $\bar{c}=K_{n} \backslash c$ is the complementary graph of $c$.

\subsection{Global and specific formulas}

Proposition 1 (see [4]). In the thermodynamic limit, the pressure of the continuous unidimensional hardcore gas model is given by

$$
\frac{P}{k T}=L(z)
$$

where $L(z)$ denotes the Lambert function, defined by the functional equation

$$
L(z) \exp (L(z))=z .
$$

Corollary 1 (see [4]). Let $n$ be an integer $\geq 1$; the total Mayer's weight $|\mathcal{C}[n]|_{w_{M}}$ of the set of all connected graphs over the set $[n]=\{1,2, \ldots, n\}$ of vertices is given by

$$
\sum_{c \in \mathcal{C}[n]} w_{M}(c)=(-n)^{n-1} .
$$

Proposition 2 (see [4]). Let $n$ be an integer $\geq 1$; the total Mayer's weight $|\mathcal{B}[n]|_{w_{M}}$ of the set of all 2 -connected graphs with $n$ vertices is given by

$$
\sum_{c \in \mathcal{B}[n]} w_{M}(c)=(-n)(n-2) !
$$

The following formula for the Mayer weight of the complete graphs $K_{n}$ was first observed from numerical results.

Proposition 3 [4] One has the following specific values of the Mayer weights: 
1. For the complete graph $K_{n}$,

$$
w_{M}\left(K_{n}\right)=(-1)^{\left(\begin{array}{l}
n \\
2
\end{array}\right)} n .
$$

2. For the (unoriented) cycle $C_{n}$ with $n$ vertices,

$$
w\left(C_{n}\right)=\frac{(-1)^{n}}{(n-1) !} \sum_{i=0}^{\left\lfloor\frac{n-1}{2}\right\rfloor}(-1)^{i}\left(\begin{array}{l}
n \\
i
\end{array}\right)(n-2 i)^{n-1} .
$$

3. For $n \geq 3$, let $K_{n} \backslash$ e denote the complete graph on $n$ vertices from which an arbitrary edge has been removed. Then

$$
w_{M}\left(K_{n} \backslash e\right)=(-1)^{\left(\begin{array}{c}
n \\
2
\end{array}\right)-1}\left(n+\frac{2}{(n-1)}\right) .
$$

\subsection{The Ehrhart polynomial}

Except for the sign, the weight

$$
w_{M}(c)=(-1)^{e(c)} \int_{\mathbb{R}^{n-1}} \prod_{\{i, j\} \in c} \chi\left(\left|x_{i}-x_{j}\right|<1\right) d x_{1} \ldots d x_{n-1}, \quad x_{n}=0,
$$

can be seen as the volume of a convex polytope $\mathcal{P}(c)$ in $\mathbb{R}^{n}$ bounded by the constraints $\left|x_{i}-x_{j}\right| \leq 1$, for $\{i, j\} \in c$, with $x_{n}=0$. We can compute this volume using Ehrhart polynomials (see [10]).

Proposition 4 (Ehrhart). Let $\mathcal{P}$ be a convex polytope of dimension $d$ in $\mathbb{R}^{m}$, with vertices having integer coordinates. Let $k \mathcal{P}=\{k \alpha: \alpha \in \mathcal{P}\}$ denote the $k$-fold expansion of $\mathcal{P}$, and $I(\mathcal{P}, k)$, the number of points with integer coordinates which lie inside $k \mathcal{P}$. Then $I(\mathcal{P}, k)$ is a polynomial function of $k$ of degree $d$ whose leading coefficient is the volume $\operatorname{Vol}(\mathcal{P})$ of $\mathcal{P}$.

Proposition 5 [4]. Let $c$ be a connected graph with its $n$ vertices labelled $\{1,2, \ldots, n\}$, and define the convex polytope $\mathcal{P}(c) \subset \mathbb{R}^{n}$ by

$$
\mathcal{P}(c)=\left\{X \in \mathbb{R}^{n} \mid x_{n}=0 \quad \text { and } \quad\left|x_{i}-x_{j}\right| \leq 1 \quad \forall\{i, j\} \in c\right\},
$$

where $X=\left(x_{1}, \ldots, x_{n}\right)$. Then the vertices of $\mathcal{P}(c)$ have integer coordinates.

Using Ehrhart polynomials, we have been able to compute the weights $w_{M}(b)$ and $w_{R H}(b)$ for all 2connected graphs of size 7 and 8 and we are able to compute the weights $w_{R H}(b)$ for any 2-connected graphs of size up to 13. A table for sizes up to 8 is available from the authors. See [3].

\subsection{Graph homomorphisms}

To evaluate the volume of the polytope $\mathcal{P}(c)$ we can decompose it into $\nu(c)$ subpolytopes which are all simplices of volume $1 /(n-1)$ ! (see [5]). We obtain these subpolytopes by fixing the integral parts and the relative positions of the fractional parts of the coordinates $x_{1}, \ldots, x_{n}$ of points $X \in \mathcal{P}(c)$. The number of such configurations will then yield $\nu(c)$ and we will have $\operatorname{Vol}(\mathcal{P}(c))=\nu(c) /(n-1)$ !.

In fact, to each real number $x$, we can associate an ordered pair $\left(\xi_{x}, h_{x}\right)$ where $h_{x}=\lfloor x\rfloor$ is the integral part of $x$ and $\xi_{x}=x-h_{x}$ is the (positive) fractional part of $x$, so that $x=\xi_{x}+h_{x}$. Then, the condition 
$|x-y|<1$ translates into " $\xi_{x} \neq \xi_{y}$ and assuming $\xi_{x}<\xi_{y}$, then $h_{x}=h_{y}$ or $h_{x}=h_{y}+1$ ". Geometrically, the slope of the line segment between $x$ and $y$ should be either null or negative. Now consider a connected graph $c$ with vertex set $V=[n]=\{1,2, \ldots, n\}$, and let $X=\left(x_{1}, \ldots, x_{n}\right)$ be a point in the polytope $\mathcal{P}(c)$. Let us write $\left(\xi_{i}, h_{i}\right)$ for the fractional representation of the coordinate $x_{i}$ of $X, i=1, \ldots, n$. For $x_{n}=0$, it will be convenient to use the special representation $\xi_{n}=1.0$ and $h_{n}=-1$. The volume of $\mathcal{P}(c)$ is not changed by removing all hyperplanes $\left\{x_{i}-x_{j}=k\right\}$, for $k \in \mathbb{Z}$. Hence, we can assume that all the fractional parts $\xi_{i}$ are distinct. We form a subpolytope of $\mathcal{P}(c)$ by keeping the "heights" $h_{1}, h_{2}, \ldots, h_{n}$ fixed as well as the relative positions (total order) of the fractional parts $\xi_{1}, \xi_{2}, \ldots, \xi_{n}$. Let $h: V \rightarrow \mathbb{Z}$ denote the height function $i \mapsto h_{i}$ and $\beta: V \rightarrow[n]$ be the permutation of $[n]$ for which $\beta(i)$ gives the rank of $\xi_{i}$ in this total order. Note that $\beta(n)=n$. For example, if $n=5$ and $\xi_{3}<\xi_{4}<\xi_{2}<\xi_{1}<\xi_{5}$, then $\beta(1)=4, \beta(2)=3, \beta(3)=1, \beta(4)=2$ and $\beta(5)=5$, i.e. $\beta=(4,3,1,2,5)$. The corresponding subpolytope will be denoted by $\mathcal{P}(h, \beta)$. Let us choose a canonical point $X=X_{h, \beta}$ of $\mathcal{P}(h, \beta)$, say the centroid, obtained by setting $\xi_{i}=\beta(i) / n, i=1, \ldots, n$. Using the fractional coordinates to represent this canonical point $X_{h, \beta}$ of $\mathcal{P}(h, \beta)$, and drawing a dotted line segment between $x_{i}$ and $x_{j}$ for each edge $i, j$ of the graph $c$, we obtain a configuration in the plane which can be seen as an homomorphic image of $c$ and which characterizes the subpolytope $\mathcal{P}(h, \beta)$.

Proposition $6([4])$. Let c be a connected graph with vertex set $V=[n]$ and consider a function $h: V \rightarrow$ $\mathbb{Z}$ and a bijection $\beta: V \rightarrow[n]$ satisfying $\beta(n)=n$. Then the pair $(h, \beta)$ determines a valid subpolytope $\mathcal{P}(h, \beta)$ of $\mathcal{P}(c)$ if and only if the following condition is satisfied:

$$
\text { for any edge }\{i, j\} \text { of } c, \beta(i)<\beta(j) \text { implies } h_{i}=h_{j} \text { or } h_{i}=h_{j}+1 \text {. }
$$

Proposition 7 ([4]). Let c be a connected graph and let $\nu(c)$ be the number of pairs $(h, \beta)$ such that the condition 20 is satisfied. Then the volume of the polytope $\mathcal{P}(c)$ defined by 19$]$ is given by

$$
\operatorname{Vol}(\mathcal{P}(c))=\nu(c) /(n-1) !
$$

Proposition 7 can be used to compute the weight of some families of graphs, since $w_{M}(c)=(-1)^{e(c)} \operatorname{Vol}(\mathcal{P}(c))$.

\section{Some new explicit formulas}

Here are some of our results concerning explicit formulas for certain infinite families of graphs. These were first conjectured from numerical results using Ehrhart polynomials. Their proof uses the techniques of graph homorphisms. The weights are given in absolute value $|w(c)|$, the sign being always equal to $(-1)^{e(c)}$.

Proposition 8 For $n \geq 3$, let $K_{n} \backslash$ e denote the complete graph on $n$ vertices from which an arbitrary edge has been removed. Then we have

$$
\left|w_{R H}\left(K_{n} \backslash e\right)\right|=\frac{2}{(n-1)} .
$$

Let $S_{k}$ denote the $k$-star graph i.e., with vertex set $[k+1]$ and edge set $\{\{1,2\},\{1,3\}, \ldots,\{1, k+1\}\}$.

Proposition 9 For $n \geq 6$, let $g_{n}=K_{n} \backslash S_{3}$, then we have

$$
\left|w_{M}\left(g_{n}\right)\right|=n+\frac{6}{(n-1)}+\frac{12}{(n-1)(n-3)}, \quad\left|w_{R H}\left(g_{n}\right)\right|=\frac{12}{(n-1)(n-2)(n-3)} .
$$


Proposition 10 For $n \geq 7$, let $g_{n}=K_{n} \backslash S_{4}$, then we have

$$
\begin{gathered}
\left|w_{M}\left(g_{n}\right)\right|=n+\frac{8}{(n-1)}+\frac{24}{(n-1)(n-2)}+\frac{48}{(n-1)(n-2)(n-4)}, \\
\left|w_{R H}\left(g_{n}\right)\right|=\frac{48}{(n-1)(n-2)(n-3)(n-4)} .
\end{gathered}
$$

More generally:

Proposition 11 For $k \geq 1, n \geq k+3$, let $g_{n}=K_{n} \backslash S_{k}$, then we have

$$
\left|w_{M}\left(g_{n}\right)\right|=n+2 \sum_{j=1}^{k} \frac{j !\left(\begin{array}{c}
k \\
j
\end{array}\right)}{(n-1)(n-2) \cdots(n-j)}, \quad\left|w_{R H}\left(g_{n}\right)\right|=\frac{2 k !}{(n-1)(n-2) \cdots(n-k)} .
$$
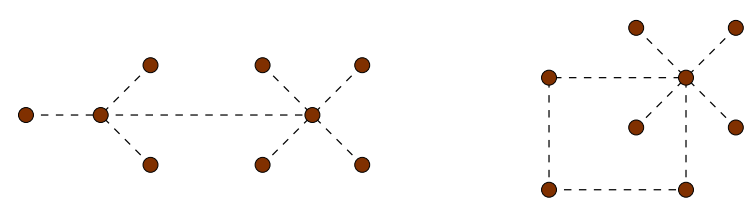

b)

Fig. 1: a) the graph $\left(S_{3}-S_{4}\right) ;$ b) the graph $\left(C_{4} \cdot S_{4}\right)$

Let $\left(S_{j}-S_{k}\right)$ denote the graph obtained by joining with a new edge the centers of a $j$-star and of a $k$-star. See Figure 1 1 ) for an example.

Proposition 12 For $n \geq 6$, let $g_{n}=K_{n} \backslash\left(S_{1}-S_{2}\right)$, then we have

$$
\begin{gathered}
\left|w_{M}\left(g_{n}\right)\right|=n+\frac{8}{(n-1)}+\frac{16}{(n-1)(n-2)}+\frac{16}{(n-1)(n-2)(n-3)}+\frac{4}{(n-1)(n-2)(n-3)(n-4)} \\
\left|w_{R H}\left(g_{n}\right)\right|=\frac{4}{(n-1)(n-2)(n-3)(n-4)}
\end{gathered}
$$

Proposition 13 For $n \geq 7$, let $g_{n}=K_{n} \backslash\left(S_{1}-S_{3}\right)$, then we have

$$
\left|w_{R H}\left(g_{n}\right)\right|=\frac{12}{(n-1)(n-2)(n-3)(n-4)(n-5)} .
$$

More generally:

Proposition 14 For $k \geq 1, n \geq k+4$, let $g_{n}=K_{n} \backslash\left(S_{1}-S_{k}\right)$, then we have

$$
\left|w_{R H}\left(g_{n}\right)\right|=\frac{2 k !}{(n-1)(n-2) \cdots(n-(k+2))} .
$$


Proposition 15 Let $g_{n}=K_{n} \backslash\left(S_{2}-S_{2}\right)$, then we have

$$
\begin{aligned}
& \left|w_{M}\left(g_{n}\right)\right|=n+\frac{10}{(n-1)}+\frac{24}{(n-1)(n-2)}+\frac{32}{(n-1)(n-2)(n-3)}+\frac{16}{(n-1)(n-2)(n-3)(n-4)} \\
& +\frac{8}{(n-1)(n-2)(n-3)(n-4)(n-5)}, n \geq 7 ; \quad\left|w_{M}\left(g_{6}\right)\right|=10 \text {. } \\
& \left|w_{R H}\left(g_{n}\right)\right|=\frac{8}{(n-1)(n-2)(n-3)(n-4)(n-5)}, n \geq 7 ; \quad\left|w_{R H}\left(g_{6}\right)\right|=\frac{2}{15} .
\end{aligned}
$$

Proposition 16 Let $g_{n}=K_{n} \backslash\left(S_{2}-S_{3}\right)$, then we have

$$
\left|w_{R H}\left(g_{n}\right)\right|=\frac{24}{(n-1)(n-2)(n-3)(n-4)(n-5)(n-6)}, n \geq 8 ; \quad\left|w_{R H}\left(g_{7}\right)\right|=\frac{1}{15} .
$$

More generally:

Proposition 17 For $k \geq 1, n \geq k+5$, let $g_{n}=K_{n} \backslash\left(S_{2}-S_{k}\right)$, then we have

$$
\left|w_{R H}\left(g_{n}\right)\right|=\frac{4 k !}{(n-1)(n-2) \cdots(n-(k+3))} .
$$

Proposition 18 For $k \geq 1, j \geq 1, n \geq k+j+3$, let $g_{n}=K_{n} \backslash\left(S_{j}-S_{k}\right)$, then we have

$$
\left|w_{R H}\left(g_{n}\right)\right|=\frac{2 k ! j !}{(n-1)(n-2) \cdots(n-(k+j+1))} .
$$

Proposition 19 For $n \geq 6$, let $g_{n}=K_{n} \backslash C_{4}$, where $C_{4}$ is the unoriented cycle with 4 vertices then we have

$$
\begin{gathered}
\left|w_{M}\left(g_{n}\right)\right|=n+\frac{8}{(n-1)}+\frac{16}{(n-1)(n-2)}+\frac{16}{(n-1)(n-2)(n-3)}, \\
\left|w_{R H}\left(g_{n}\right)\right|=\frac{8}{(n-1)(n-2)(n-3)} .
\end{gathered}
$$

Let $\left(C_{4} \cdot S_{k}\right)$ denote the graph obtained by identifying one vertex of the graph $C_{4}$ with the center of a $k$-star. See Figure $1 \mathrm{p}$ ) for an example.

Proposition 20 For $n \geq 6$, let $g_{n}=K_{n} \backslash\left(C_{4} \cdot S_{1}\right)$, then we have

$$
\begin{gathered}
\left|w_{M}\left(g_{n}\right)\right|=n+\frac{10}{(n-1)}+\frac{24}{(n-1)(n-2)}+\frac{32}{(n-1)(n-2)(n-3)}+\frac{12}{(n-1)(n-2)(n-3)(n-4)}, \\
\left|w_{R H}\left(g_{n}\right)\right|=\frac{4}{(n-1)(n-2)(n-3)(n-4)} .
\end{gathered}
$$

Proposition 21 For $n \geq 7$, let $g_{n}=K_{n} \backslash\left(C_{4} \cdot S_{2}\right)$, then we have

$$
\left|w_{R H}\left(g_{n}\right)\right|=\frac{8}{(n-1)(n-2)(n-3)(n-4)(n-5)} .
$$


More generally:

Proposition 22 For $k \geq 1, n \geq k+5$, let $g_{n}=K_{n} \backslash\left(C_{4} \cdot S_{k}\right)$, then we have

$$
\left|w_{R H}\left(g_{n}\right)\right|=\frac{4 k !}{(n-1)(n-2) \cdots(n-(k+3))} .
$$

Let $P_{k}$ denote the path graph i.e., the graph with vertex set $[k]$ and edges set $\{\{1,2\},\{2,3\}, \ldots,\{k-$ $1, k\}\}$.

Proposition 23 For $n \geq 5$, let $g_{n}=K_{n} \backslash P_{3}$, then we have

$$
\left|w_{M}\left(g_{n}\right)\right|=n+\frac{4}{(n-2)}, \quad\left|w_{R H}\left(g_{n}\right)\right|=\frac{4}{(n-1)(n-2)} .
$$

Proposition 24 For $n \geq 5$, let $g_{n}=K_{n} \backslash P_{4}$, then we have

$\left|w_{M}\left(g_{n}\right)\right|=n+\frac{6}{(n-1)}+\frac{8}{(n-1)(n-2)}+\frac{2}{(n-1)(n-2)(n-3)},\left|w_{R H}\left(g_{n}\right)\right|=\frac{2}{(n-1)(n-2)(n-3)}$.

Proposition 25 For $k \geq 5, n \geq k+1$, let $g_{n}=K_{n} \backslash P_{k}$, then we have

$$
\left|w_{M}\left(g_{n}\right)\right|=n+\frac{2(k-1)}{(n-1)}+\frac{4(k-2)}{(n-1)(n-2)}+\frac{2(k-3)}{(n-1)(n-2)(n-3)}, \quad w_{R H}\left(g_{n}\right)=0 .
$$

\section{Some physical background}

\subsection{The grand canonical partition function}

In the context of a non-ideal gas with $n$ particles in a vessel $V$ included in $\mathbb{R}^{d}$, the particle positions are given by vectors $\overrightarrow{x_{1}}, \overrightarrow{x_{2}}, \ldots, \overrightarrow{x_{n}}$. If we assume that the system is free from external influences, the partition function is defined as

$$
Z(V, T, n)=\frac{1}{n ! \lambda^{d n}} \int_{V} \cdots \int_{V} \exp \left(-\beta \sum_{i<j} \varphi\left(\left|\overrightarrow{x_{i}}-\overrightarrow{x_{j}}\right|\right)\right) d \overrightarrow{x_{1}} \cdots d \overrightarrow{x_{n}}
$$

where $\lambda$ and $\beta$ depend on the temperature $T$ and where the interaction between two particles at distance $r$ is expressed by a potential function $\varphi(r)$ as illustrated in Figure 2 a).

The grand canonical partition function is defined as the generating series of the partition functions:

$$
Z_{\mathrm{gr}}(V, T, z)=\sum_{n=0}^{\infty} Z(V, T, n)\left(\lambda^{d} z\right)^{n}
$$

where $z$ is called the fugacity or the activity of the system. The generating function identities are in the sense of formal power series in the activity $z$. The system's macroscopic parameters can be described using the grand canonical partition function. In particular, the pressure $P$, the mean number of particles $\bar{n}$ and the density $\rho$ can be written as

$$
\frac{P}{k T}=\frac{1}{V} \log Z_{\mathrm{gr}}(V, T, z), \bar{n}=z \frac{\partial}{\partial z} \log Z_{\mathrm{gr}}(V, T, z) \quad \text { and } \quad \rho=\frac{\bar{n}}{V},
$$

where $V$ is also used as the volume of the vessel. 

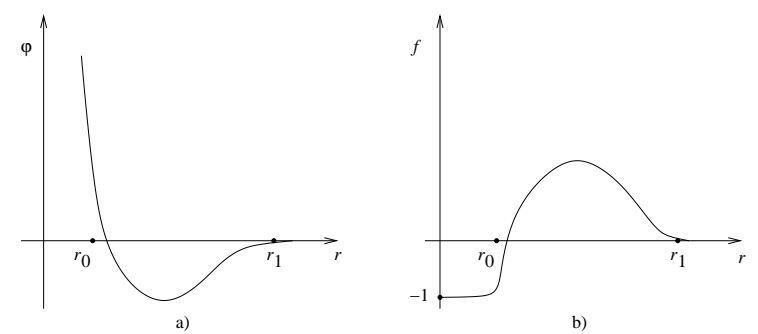

Fig. 2: a) the function $\varphi(r)$; b) the function $f(r)$

\subsection{Mayer's idea}

In order to study these functions, Mayer (1940) sets

$$
f_{i j}=\exp \left(-\beta \varphi\left(\left|\overrightarrow{x_{i}}-\overrightarrow{x_{j}}\right|\right)\right)-1,
$$

where $f_{i j}=f\left(\left|\overrightarrow{x_{i}}-\overrightarrow{x_{j}}\right|\right)$. The general form of Mayer's function

$$
f(r)=\exp (-\beta \varphi(r))-1,
$$

compared to the potential function $\varphi(r)$, is shown in Figure 2 . Since

$$
\prod_{1 \leq i \leq j \leq n}\left(1+f_{i j}\right)=\sum_{g \in \mathcal{G}[n]} \prod_{\{i, j\} \in g} f_{i j},
$$

where $\mathcal{G}[n]$ denotes the set of all simple graphs over the set of vertices $[n]=\{1,2, \ldots, n\}$, the partition function becomes

$$
\begin{aligned}
Z(V, T, n) & =\frac{1}{n ! \lambda^{d n}} \int_{V} \cdots \int_{V} \exp \left(-\beta \sum_{i<j} \varphi\left(\left|\overrightarrow{x_{i}}-\overrightarrow{x_{j}}\right|\right)\right) d \overrightarrow{x_{1}} \cdots d \overrightarrow{x_{n}} \\
& =\frac{1}{n ! \lambda^{d n}} \sum_{g \in \mathcal{G}[n]} W_{M}(g)
\end{aligned}
$$

where the weight $W_{M}(g)$ of a graph $g$ is given by the integral

$$
W_{M}(g)=\int_{V} \cdots \int_{V} \prod_{\{i, j\} \in g} f_{i j} d \overrightarrow{x_{1}} \cdots d \overrightarrow{x_{n}} .
$$

This is the First Mayer weight of a graph $g$. In terms of $W_{M}(g)$, the grand-canonical function becomes

$$
\begin{aligned}
Z_{\mathrm{gr}}(V, T, z) & =\sum_{n=0}^{\infty} Z(V, T, n)\left(\lambda^{d} z\right)^{n}=\sum_{n=0}^{\infty} \frac{1}{n !} \sum_{g \in \mathcal{G}[n]} W_{M}(g) z^{n} \\
& =\mathcal{G}_{W_{M}}(z)
\end{aligned}
$$

the exponential generating function of the species $\mathcal{G}$ of graphs weighted by the function $W_{M}$. 
Proposition 26 (see [4]). The First Mayer weight of a simple graph $g W_{M}(g)$ is multiplicative on the connected components $c_{k}$ of $g, 1 \leq k \leq m: W_{M}(g)=W_{M}\left(c_{1}\right) W_{M}\left(c_{2}\right) \cdots W_{M}\left(c_{m}\right)$.

Corollary 2 (see [4]). The pressure of the system can be expressed in terms of the exponential generating function of the species $\mathcal{G}$ of connected graphs weighted by $W_{M}$. More precisely, we have

$$
\frac{P}{k T}=\frac{1}{V} \log Z_{g r}(V, T, z)=\frac{1}{V} \log \mathcal{G}_{W_{M}}(z)=\frac{1}{V} \mathcal{C}_{W_{M}}(z) .
$$

\subsection{The thermodynamic limit $w_{M}(c)$}

Let $c$ be a connected graph over $\{1,2, \ldots, n\}$. The second Mayer weight $w_{M}(c)$ is defined as the thermodynamic limit

$$
w_{M}(c)=\lim _{V \longrightarrow \infty} \frac{1}{V} W_{M}(c)=\lim _{V \longrightarrow \infty} \frac{1}{V} \int_{V} \cdots \int_{V} \prod_{\{i, j\} \in c} f_{i j} d \overrightarrow{x_{1}} \cdots d \overrightarrow{x_{n}}
$$

Here, $V$ going to infinity has the following meaning. The vessel $V \in \mathbb{R}^{d}$ must contain a ball $B(0, R)$ centered at the origin, with radius $R \in] 0, \infty) . V$ goes to infinity means that $R$ goes to infinity. The following proposition gives us the conditions on Mayer's function $f$ for $w_{M}(c)$ to exist.

Proposition 27 (see [4]). If the function $f:[0, \infty) \rightarrow \mathbb{R}$ is integrable and bounded and if $\int_{0}^{\infty} r^{d-1}|f(r)| d r$ $<\infty$, then for any fixed $\vec{x}_{n} \in \mathbb{R}^{d}$, the function $F_{\vec{x}_{n}}: \mathbb{R}^{d \cdot(n-1)} \rightarrow \mathbb{R}$, defined by $F_{\overrightarrow{x_{n}}}\left(\overrightarrow{x_{1}}, \ldots, \overrightarrow{x_{n-1}}\right)=$ $\prod_{\{i, j\} \in c} f\left(\left|\overrightarrow{x_{i}}-\overrightarrow{x_{j}}\right|\right)=\prod_{\{i, j\} \in c} f_{i j}$ is integrable over $\left(\mathbb{R}^{d}\right)^{n-1}$ and its integral is independent of $\overrightarrow{x_{n}}$. Moreover the limit 55) exists and is equal to

$$
w_{M}(c)=\int_{\left(\mathbb{R}^{d}\right)^{n-1}} \prod_{\{i, j\} \in c} f\left(\left\|\overrightarrow{x_{i}}-\overrightarrow{x_{j}}\right\|\right) d \overrightarrow{x_{1}} \cdots d \overrightarrow{x_{n-1}}, \quad \overrightarrow{x_{n}}=0 .
$$

Proposition 28 (see [4]). In the thermodynamic limit, the pressure of the system is given directly in terms of the exponential generating function of connected graphs weighted by the Second Mayer Weight $w_{M}(c)$ :

$$
\frac{P}{k T}=\mathcal{C}_{w_{M}}(z)
$$

Proposition 29 (see [4]). The second Mayer weight $w_{M}$ is block-multiplicative. More precisely, for any connected graph $c$ whose blocks are $b_{1}, b_{2}, \ldots, b_{m}$, we have

$$
w_{M}(c)=w_{M}\left(b_{1}\right) w_{M}\left(b_{2}\right) \ldots w_{M}\left(b_{m}\right),
$$

where a block in a connected graph is a maximal 2-connected subgraph.

\subsection{The virial expansion}

In order to better explain the thermodynanic behaviour of non ideal gases, Kamerlingh Onnes proposed, in 1901, a series expansion of the form

$$
\frac{P}{k T}=\rho+\beta_{2} \rho^{2}+\beta_{3} \rho^{3}+\cdots,
$$


where $\rho=\frac{\bar{n}}{V}$ is the density, called the virial expansion. A benefit of Mayer's theory is a formal derivation of this expansion and an interpretation of the virial coefficients $\beta_{n}, n \geq 2$, as the total weight of the set $\mathcal{B}[n]$ of all labelled 2-connected graphs over the set $[n]$ of vertices (see [6] and [11]). More precisely

$$
\begin{aligned}
\beta_{n} & =\frac{1-n}{n !}|\mathcal{B}[n]|_{w_{M}} \\
& =\frac{1-n}{n !} \sum_{b \in \mathcal{B}[n]} w_{M}(b) \\
& =\frac{1-n}{n !} \sum_{b \in T\left(\mathcal{B}_{n}\right)} \ell(b) w_{M}(b),
\end{aligned}
$$

where the summation in 60 is taken over a set of representatives of the set $T\left(\mathcal{B}_{n}\right)$ of isomorphism types of 2-connected graphs with $n$ vertices, and $\ell(b)$ is the number of labellings of the graph $b$.

\subsection{The Ree-Hoover expansion}

An important rewriting of the virial coefficients was performed by Ree and Hoover ([7], [8]) by introducing the function

$$
\bar{f}(r)=1+f(r),
$$

and then expanding each weight $w_{M}(b)$ by substituting $1=\bar{f}-f$ for pairs of vertices not connected by edges. Upon performing this rewriting of the Mayer weights, vertices in the resultant graphs will all be mutually connected by either $f$ bonds or $\bar{f}$ bonds. Now there is a new factor which appears for each graph, called the star content by Ree and Hoover [8], which may be either positive or negative. For example, we have

$$
\begin{aligned}
& w_{M}(\searrow)=w_{R H}(\searrow), \\
& w_{M}(\longmapsto)=w_{R H}(\longmapsto)-w_{R H}(\searrow), \\
& w_{M}\left(\lfloor)=w_{M R H}\left((\vdots)-w_{M}(\longmapsto)\right.\right.
\end{aligned}
$$

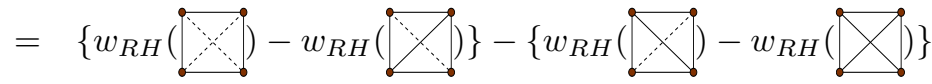

$$
\begin{aligned}
& =w_{R H}(\ggg)-2 \cdot w_{R H}(\longleftrightarrow)+w_{R H}(\Longleftrightarrow) \text {. }
\end{aligned}
$$

Now using the three equations 662, ,63, $, 664,|\mathcal{B}[4]|_{w_{M}}$ may be written as

$$
\begin{aligned}
& |\mathcal{B}[4]|_{w_{M}}=1 \cdot w_{M}(\)+6 \cdot w_{M}(\bigvee)+3 \cdot w_{M}(\square) \\
& =(-2) \cdot w_{R H}(\bigotimes)+0 \cdot w_{R H}(\)+3 \cdot w_{R H}(\Longleftrightarrow)
\end{aligned}
$$

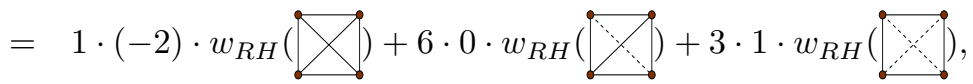


where (-2), 0, 1 are the star contents for the graphs have

$$
|\mathcal{B}[n]|_{w_{M}}=\sum_{b \in \mathcal{B}[n]} \bar{a}_{n}(b) w_{R H}(b),
$$

where the coefficient $\bar{a}_{n}(b)$ is the star content of the graph $b$, and $w_{R H}(b)$ is the Ree-Hoover weight of the graph $b$ :

$$
w_{R H}(b)=\lim _{V \longrightarrow \infty} \frac{1}{V} \int_{V} \cdots \int_{V} \prod_{\{i, j\} \in b} f_{i j} \prod_{\{i, j\} \in \bar{b}} \bar{f}_{i j} d \overrightarrow{x_{1}} \cdots d \overrightarrow{x_{n}} .
$$

However, when the Ree-Hoover transformation is made many graphs have zero star content and hence do not contribute to the virial coefficient. In addition some Ree-Hoover graph weights may be zero for geometrical reasons.

\section{References}

[1] Nathan Clisby and Barry M. McCoy, "Negative virial coefficients and the dominance of loose packed diagrams for D-dimensional hard spheres.” Journal of Statistical Physics, 114 (2004), 1361-1392.

[2] Nathan Clisby and Barry M. McCoy, "Ninth and tenth order virial coefficients for hard spheres in $D$ dimensions." Journal of Statistical Physics, 122 (2006), 15-57.

[3] Kaouche, A., Leroux P. "Tables for the Mayer and Ree-Hoover graph weights of 2-connected graphs of size 4 to 7." [http://www.lacim.uqam.ca/ kaouche/TableauRH7.pdf] "Tables for the Mayer and Ree-Hoover graph weights of 2-connected graphs of size 8." [http://www.lacim.uqam.ca/ kaouche/TableauRH8.pdf].

[4] Labelle G., Leroux P. and Ducharme M. G., "Graph weights arising from Mayer's theory of cluster integrals," Seminaire Lotharingien de Combinatoire 54 (2007), Article B54m.

[5] Bodo Lass, Personal communication, 2005.

[6] Pierre Leroux, "Enumerative problems inspired by Mayer's theory of cluster integrals." The Electronic Journal of Combinatorics, vol.11 (2004), R32.

[7] F. H. Ree and W. G. Hoover, "Fifth and sixth virial coefficients for hard spheres and hard discs." The Journal of Chemical Physics, 40 (1964), 939-950.

[8] F. H. Ree and W. G. Hoover, " Reformulation of the Virial Series for Classical Fluids.” The Journal of Chemical Physics, 41 (1964), 1635-1645.

[9] F. H. Ree and W. G. Hoover, "Seventh virial coefficients for hard spheres and hard discs." The Journal of Chemical Physics, 46 (1967), 4181-4196.

[10] Richard P. Stanley, Enumerative Combinatorics, vol. 1, Belmont, California: Wadsworth, 1986, 306 p.

[11] G. E. Uhlenbeck and G. W. Ford, Lectures in Statistical Mechanics, Providence, Rhode Island: American Mathematical Society, 1963, 181 p. 